Gut, 1983, 24, 421-426

\title{
Radionuclide transit studies in the detection of oesophageal dysmotility
}

\author{
J N BLACKWELL, W J HANNAN, R D ADAM, AND R C HEADING \\ From the Departments of Therapeutics and Medical Physics, Royal Infirmary, Edinburgh
}

SUMmARY A scintigraphic method is described to measure the transit of a fluid bolus through the oesophagus. Transit times in 16 normal subjects ranged from five to 15 seconds and were highly reproducible. Prolonged transit times were observed in 16 of 19 patients with known oesophageal motility disorders, and in these patients inspection of the time activity curves frequently permitted an adynamic oesophagus to be distinguished from one showing excessive incoordinate contractions. The technique was then applied prospectively to 50 patients referred for oesophageal motility studies and a comparison made between the oesophageal transit measurements and the findings on conventional oesophageal manometry. There was agreement between these tests in $42(84 \%)$ of the 50 patients. The measurement of oesophageal transit may be made quickly and safely, without causing discomfort to the patient, and it appears to be at least as sensitive as manometry in the detection of oesophageal motility disorders.

Investigation by contrast radiology and upper gastrointestinal endoscopy provides a precise diagnosis in most patients with symptoms suggestive of oesophageal disease. When such symptoms are due to oesophageal motility disorders, however, the diagnosis is often more elusive. Manometry is currently the definitive investigation whereby motility disorders may be recognised but suffers the disadvantage of causing some discomfort to the patients and demands a considerable outlay of both time and expertise in the performance of the study and in the interpretation of the recording. In consequence, a quick and reliable procedure to detect oesophageal motor dysfunction would be of considerable value.

Stimulated by these considerations we have assessed a scintigraphic technique ${ }^{1}$ using a radionuclide tracer and gamma camera for the quantitation of the rate of passage of bolus through the oesophagus. The procedure was first validated in normal subjects and in patients with previously diagnosed oesophageal motility disorders, and was then applied prospectively to 50 consecutive patients in whom an oesophageal motility disorder was suspected.

\footnotetext{
Address for correspondence: Dr R C Heading, Department of Therapeutics, Royal Infirmary, Edinburgh, EH3 9YW. Received for publication 13 August 1982
}

\section{Methods}

PATIENTS

Sixteen control subjects were studied to validate the transit measurements and to establish normal values. There were 11 men aged 19-82 years (mean 47 years) and five women aged 27-87 years (mean 45 years). None of these subjects had any symptoms or history to suggest upper gastrointestinal disease.

Nineteen patients with oesophageal motor disorders confirmed by manometry were also studied. There were 11 men aged $45-65$ years (mean 52 years) and eight women aged 36-71 (mean 53 years). All had previously undergone routine barium studies of the upper gastrointestinal tract and had also undergone endoscopy with oesophageal mucosal biopsy to exclude oesophagitis. Thirteen had diffuse oesophageal spasm, of whom nine suffered intermittent dysphagia and all had episodes of non-exertional chest pain. Routine barium radiology had shown tertiary contractions in only four of these patients, whereas on oesophageal manometry, all 13 showed repetitive non-propagated oesophageal contractions together with some normal peristalsis - the features of diffuse oesophageal spasm. ${ }^{2}$ Four patients had achalasia, which in three cases had been treated by Heller's operation and in one case by pneumatic dilatation. The other two patients both had 
dysphagia owing to an adynamic distal oesophagus, one because of scleroderma (without oesophageal stricture) and the other a non-specific oesophageal motility disorder.

Transit measurements were also performed on 50 consecutive patients referred for oesophageal manometry to investigate the possibility that oesophageal dysmotility was responsible for their symptoms of chest pain (24), dysphagia (12), chest pain + dysphagia (7), odynophagia (2), rumination (2), and vomiting (3). There were 21 women aged 17-71 years (mean 50 years) and 29 men aged $22-70$ years (mean 44 years). All had been investigated by conventional barium radiology of the upper gastrointestinal tract which was reported abnormal in only 11 cases; showing tertiary contractions (3), suggesting achalasia (1), a hiatus hernia (3), and gastro-oesophageal reflux (3). All patients had also undergone upper gastrointestinal endoscopy with biopsy to exclude oesophagitis. Transit measurements were performed and analysed by two of us (RDA and WJH) without knowledge of the clinical details, and before manometry, which was carried out within one to three hours of the transit test. Similarly, manometry was reported independently of the results of the transit test.

\section{SCINTIGRAPHIC TECHNIQUE}

After fasting for three hours, the subject was positioned supine beneath a gamma camera fitted with a high sensitivity diverging collimator. The field of view included the mouth and extended to the epigastrium. In initial studies, the gamma camera was linked to a video recorder and a recording made in list mode for subsequent replay to a computer for analysis. In the patients studied prospectively, the gamma camera was linked directly to a small, mobile computer (Ohio Nuclear MCS 560), and the data stored on computer discs.

With the patient in position, a radioactive marker was briefly placed over the cricoid cartilage to record the position of the upper end of the oesophagus. ${ }^{99 \mathrm{~m}}$ Technetium sulphur colloid, 0.5 $\mathrm{mCi}(20 \mathrm{MBq})$, was made up to $10 \mathrm{ml}$ with water and was then introduced into the mouth by syringe. The subject was asked to swallow this in a single swallow and not to swallow again until instructed to 'dry swallow' 30 seconds later and at subsequent 30 second intervals for up to two minutes. Preliminary 'rehearsal' using $10 \mathrm{ml}$ water without the isotope helped the subjects to understand what they should do. In nine of the 16 normal subjects, the isotope swallow was then repeated to assess the reproducibility of the measurement. The procedure was performed twice in all the patients with oesophageal motility disorders and in the patients studied prospectively.

On replay of the recorded swallow, the position of the cricoid marker indicated the upper end of the oesophagus and the distal end could be identified without difficulty from the position of the isotope lying in the gastric fundus after its clearance from the oesophagus. From these points, the length of the oesophagus was divided into proximal, middle, and distal thirds (Fig. 1). For analysis, 55 frames at frame rates of one per 0.3 seconds proved appropriate for normal subjects, though longer frame times up to one per 5 seconds were required for patients with delayed oesophageal clearance.

The computer was used to analyse the rate of passage of the bolus through each third of the oesophagus by the production of graphs plotting radioactivity against time in seconds. From these time-activity curves, the transit time - that is, entry to clearance of radioactivity - could be measured. In the normal controls, the transit times were measured for the proximal, middle, and distal thirds as well as the total transit time for the whole oesophagus. In the patients, we found the total transit time to be the more useful measurement and defined this as the interval between the appearance of radioactivity in the upper third of the oesophagus and the clearance of all but $10 \%$ of peak activity from the lower third.

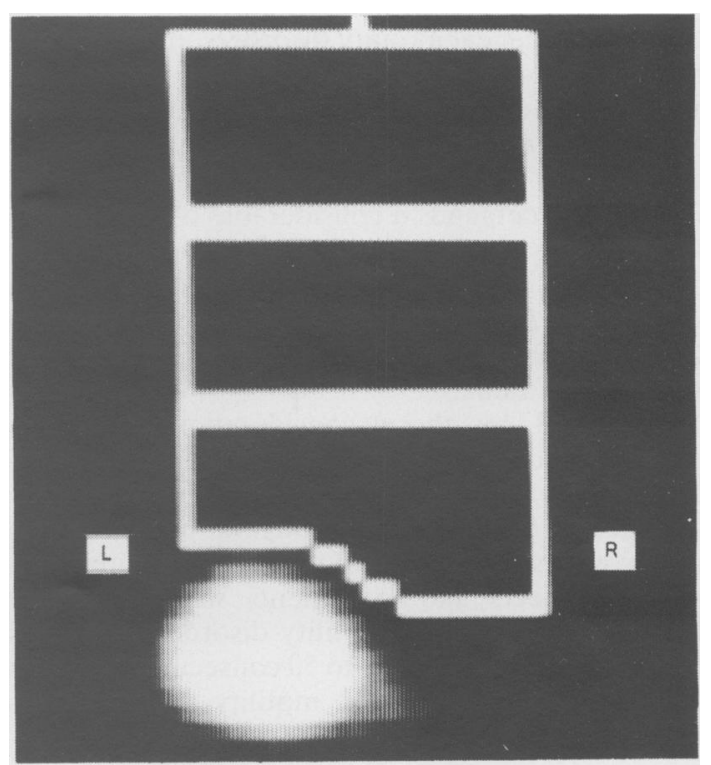

Fig. 1 Showing cricoid marker top and fundal pool of radioactivity. From these landmarks oesophagus may be divided into three areas of interest. 
We have calculated that the maximum radiation dose to the subject is $0.4 \mathrm{rad}$ per $\mathrm{mCi}$ of ${ }^{99 \mathrm{~m}}$ technetium, $(0.1 \mathrm{mGy} / \mathrm{MBq})$, to the colonic mucosa.

\section{MANOMETRIC TECHNIQUE}

Manometry was undertaken in a conventional manner, using an Arndorfer M3 8 lumen polyvinyl tube of $4.5 \mathrm{~mm}$ external diameter, each lumen having an internal diameter and side opening of 0.8 $\mathrm{mm}$. Each lumen was perfused with water at a constant rate of $0.7 \mathrm{ml} / \mathrm{min}$ from an Arndorfer capillary infusion pump. Pressure changes were detected by pressure transducers (Elcomatic EM 750) linked via amplifier modules to a chart recorder. This is a low compliance system giving a pressure rise of $182 \mathrm{~mm} \mathrm{Hg}$ per second when a side opening is occluded. The lower oesophageal sphincter pressure was measured three times in each subject by the rapid pull through technique, and an attempt was made to elicit sphincter relaxation in response to swallowing. The tube was then positioned stationary to record distal oesophageal motility. In addition to recording spontaneous activity, the effect of swallowing $5 \mathrm{ml}$ aliquots of water and of swallowing 'dry' was observed.

\section{Results}

\section{NORMAL SUBJECTS}

The transit of radioactivity through the oesophagus of one normal subject is illustrated by the timeactivity tracings from the proximal, middle, and distal thirds (Fig. 2). In this subject the total transit time was five seconds. The fluid bolus characteristically traverses the proximal third most rapidly, presumably as a result of pharyngeal propulsion, and thereafter slows it as it comes under the influence of oesophageal peristalsis. Passage through the distal third was slowest, with a slight delay usually being observed at the cardia. The total transit time for the group of normal subjects was $7 \cdot 3$ sec \pm 2.3 (mean \pm SD) with a range of five to 15 seconds. The transit times for each area of the oesophagus in the normal subjects were 3.2 $\sec \pm 1.4$ for the proximal third, $4.7 \mathrm{sec} \pm 1.4$ for the middle third and $6.8 \mathrm{sec} \pm 2.2$ for the distal third. In the nine subjects in whom the two measurements were performed, the total transit time varied by one second or less in seven subjects and only by two seconds in the other two subjects.

\section{PATIENTS WITH PREVIOUSLY DIAGNOSED}

\section{OESOPHAGEAL MOTILITY DISORDERS}

Total transit times were abnormal in 16 of the 19 patients known to have oesophageal motility

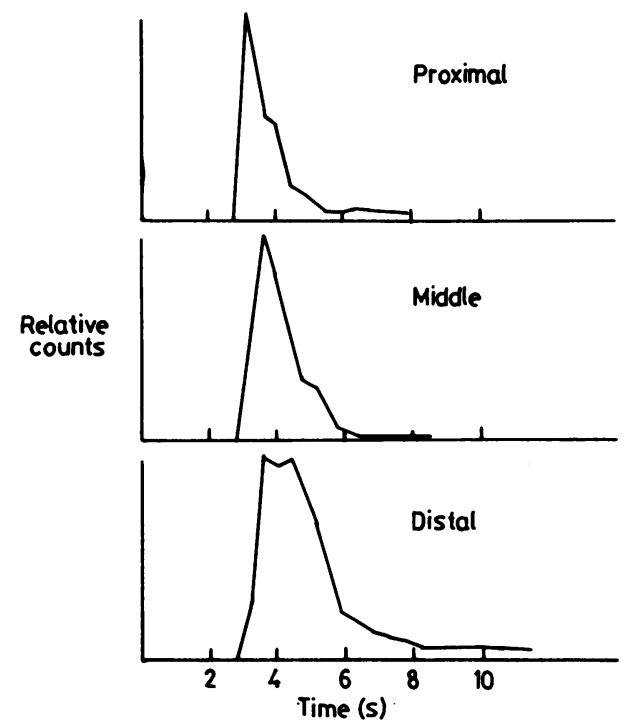

Fig. 2 Radionuclide time activity curves from normal subject. Radioactivity counts in proximal, middle, and distal thirds of oesophagus on vertical axis; time in seconds on horizontal axis.

disorders (Fig. 3). In many cases, additional information could be gained from inspection of the time-activity curves. Figure 4 shows the time activity tracing from a patient with diffuse oesophageal spasm and shows the retention of radioactivity within the middle and proximal thirds of the

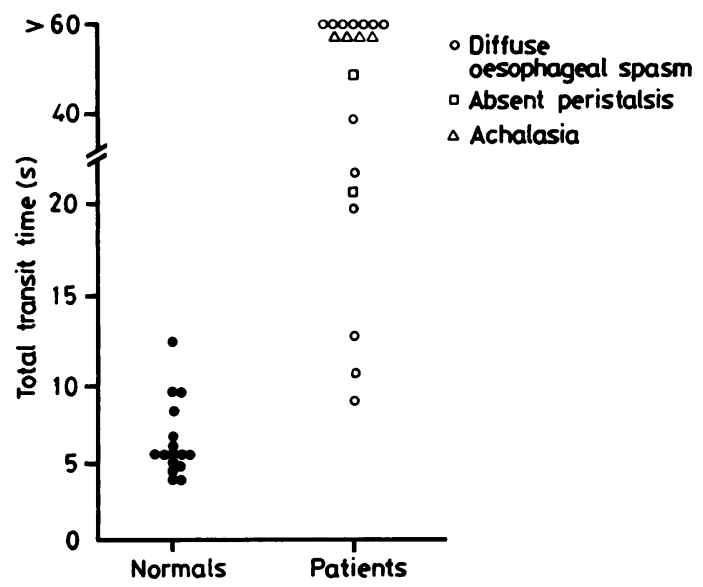

Fig. 3 Total oesophageal transit times (in seconds) for 16 normal subjects and 19 patients with oesophageal motility disorders. 


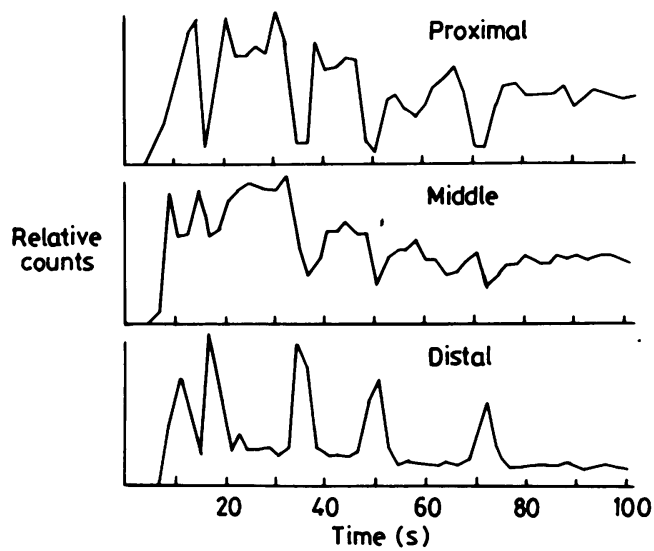

Fig. 4 Radionuclide time activity curves from patient with diffuse oesophageal spasm. Radioactivity counts in each area on vertical axis, and time in seconds on horizontal axis.

oesophagus 100 seconds after the swallow. In addition to the prolonged transit time, however, inspection of the curves shows that radioactivity was intermittently propelled proximally up the oesophagus, presumably as a consequence of incoordinate oesophageal contractions. In contrast, Fig. 5 shows a comparable record from a patient with an adynamic oesophagus (achalasia). There is a similar delay in total transit time, but much less fluctuation in the time activity curves.

Two transit measurements were made in each patient. In 14 patients, prolonged transit times were

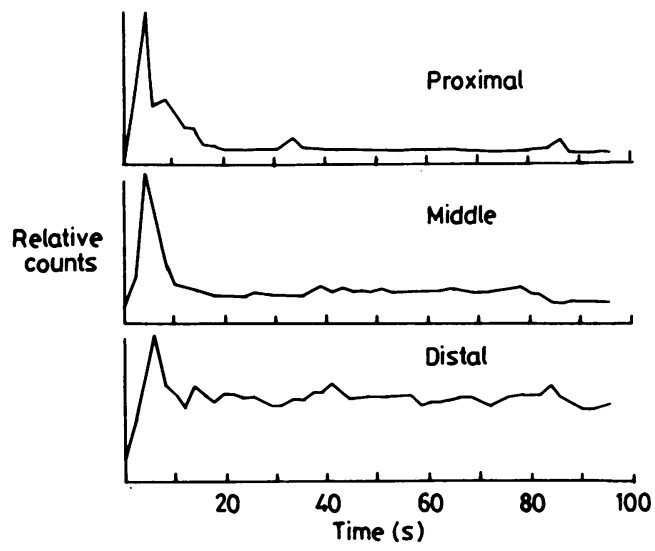

Fig. 5 Radionuclide time activity curves from patient with achalasia. Radioactivity counts in each area on vertical axis, and time in seconds in horizontal axis. recorded on both occasions while the other two patients exhibited prolonged transit on one measurement only. Three patients with diffuse spasm did not show prolongation of the transit time. These three patients experienced symptoms only intermittently, with trouble-free spells lasting for some weeks. Manometry during symptomatic phases was grossly abnormal and typical of diffuse oesophageal spasm. During asymptomatic spells, however, manometry showed only occasional nonpropagated contractions. The radionuclide transit measurements were performed during an asymptomatic period.

\section{PATIENTS STUDIED PROSPECTIVELY}

Comparison of the transit measurements and the results of oesophageal manometry in the patients studied prospectively is shown in Fig. 6. Normal manometric findings were observed in 31 patients and 26 of these had normal transit times. Nineteen patients had abnormal manometry and 16 of these had abnormal transit times. Thus the transit measurements and the manometry were in agreement in $42(84 \%)$ of these 50 patients.

Of the three patients with abnormal manometry

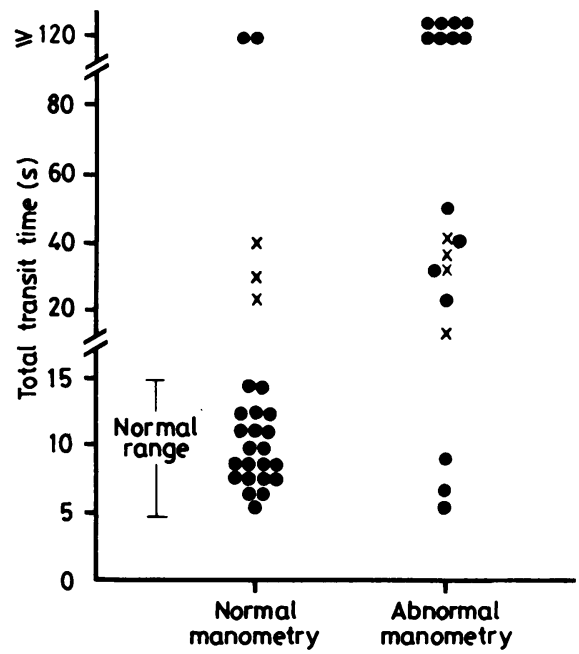

Fig. 6 Total oesophageal transit times (in seconds) for 50 patients investigated prospectively for suspected . oesophageal motility disorders. Patients have been divided into those with normal and abnormal manometry. $(\bullet)$ patients with both swallows giving transit times that were the same or within three seconds when within the normal range, or within 10 seconds when abnormal. $(x)$ patients in whom one radionuclide swallow gave normal transit time and the other an abnormal transit time. In these cases the abnormal transit time has been plotted. 
and normal transit times, one had a 'nutcracker oesophagus' - prolonged peristaltic waves sometimes exceeding $250 \mathrm{~mm} \mathrm{Hg}$, but there were no non-propagated contractions. The second patient had some very low pressure peristaltic contractions (32 $\mathrm{mm} \mathrm{Hg}$ ) but often swallowing failed to generate any contraction at all. The third patient had the manometric features of diffuse oesophageal spasm. Of the five patients with prolonged transit but normal manometry, four had unexplained intermittent chest pain and one suffered retrosternal discomfort with vomiting during or immediately after meals. Three of the patients exhibited delayed transit on only one of the two swallows, while the other two patients had markedly abnormal transit on both swallows. All five patients presently remain symptomatic without any satisfactory explanation of their complaints.

\section{Discussion}

The results of this investigation indicate that the measurement of oesophageal radionuclide transit is a sensitive means of detecting oesophageal motility disorders. The technique we have used is essentially that described by Russell ${ }^{1}$ and there are important differences from the techniques described previously by other authors. ${ }^{3-6} \mathrm{Kazem}^{3}$ was the first to describe the use of radionuclides for oesophageal transit measurements, but limited his study to normal subjects and patients who had obstructing lesions of the oesophagus. Bosch ${ }^{4}$ described a similar measurement in patients with oesophageal obstruction based on the use of radionuclide impregnated gelatin. Mayron ${ }^{5}$ briefly described the use of more than one area of interest in the oesophagus, but did not assess the technique in groups of patients. Tolin ${ }^{6}$ conducted oesophageal transit measurements in patients with oesophageal motor disorders but used only one area of interest.

Russell's technique, which we have used, differs from previous reports by examining the patients in the supine position to exclude the effects of gravity, and by analysis of data with the oesophagus divided into three areas of interest. While determination of total transit time requires a single area of interest only, the use of multiple areas of interest often permits a distinction to be drawn between an adynamic oesophagus and incoordinate activity typical of diffuse oesophageal spasm. The inclusion of the mouth in the field of view is essential to permit recognition of any occasion on which the radionuclide bolus does not leave the mouth in a single swallow. We believe that these refinements of the technique are essential for a useful and reliable interpretation of the results.
Persuasive evidence of the reproducibility of the method is provided by comparison of Russell's data with our own findings. The mean total transit time for his normal control subjects was 7.2 sec \pm 1.7 (SD) with a range of five to 11.5 seconds. These figures are very similar to those of our normal subjects, which are $7.3 \mathrm{sec} \pm 2.3$ (SD) with a range of five to 15 seconds. Our normal subjects spanned a greater age range and interestingly there was no evidence of any increase in the transit times with the age of the subjects. Russell and his colleagues studied 15 patients with manometrically confirmed oesophageal motility disorders and oesophageal transit times were abnormal in all cases. This contrasts with our own results in which three of 13 patients with previously diagnosed diffuse oesophageal spasm had normal transit times on both occasions. A further two patients had normal transit times on one of the two studies. We believe that our findings are predictable in view of the frequently intermittent nature of oesophageal spasm, as the incoordinate activity may be interspersed with normal peristaltic waves. We would, therefore, expect that any measurement based on a single swallow would, on occasions, give a normal result.

The clinical value of any new investigation must be proved by prospective studies. The finding of an $84 \%$ concordance between the results of transit measurement and oesophageal manometry is encouraging. Five patients had a prolonged transit time but normal manometry. While it is possible that the transit test has genuine false positives, it is equally possible that the test may be more sensitive than manometry. In addition, in some patients a motility abnormality may be intermittent. Three patients had abnormal manometry but normal transit times - explicable in one case by the very intermittent nature of his oesophageal spasm at manometry. In the second patient, 'giant' peristaltic waves, which were undoubtedly abnormal, would not be expected to impede the passage of a fluid bolus. The third patient had infrequent and poor amplitude peristalsis.

Patients with oesophagitis were excluded from the present study and we have not evaluated the transit measurement in patients with symptoms attributable to gastro-oesophageal reflux. The use of a gamma camera to detect and quantify reflux has been reported by others. ${ }^{7}$ In the subjects we have studied, however, episodes of gastro-oesophageal reflux were occasionally observed after initial oesophageal clearance and on such occasions, the reflux was recognisable as a sequential movement of radioactivity from the stomach into the oesophagus. The time activity tracings were readily distinguished from those showing delayed transit. 
Oesophageal transit measurements are highly acceptable to both patients and their medical attendants in terms of safety, comfort, brevity and cost. The relative simplicity of the technique should place it within the scope of any department with access to a gamma camera with data processing facilities, and a direct link of the camera to a small computer, such as we have used, allows the study to be performed and the results to be obtained within a total time of 10 minutes. The extent to which the transit measurement might legitimately replace manometry in the investigation of suspected oesophageal dysmotility remains to be determined. For physicians without ready access to manometry, its advantages over conventional radiology are clear but when both transit measurements and manometry are available, it may be that performance of both investigations will permit recognition of a higher proportion of patients with oesophageal dysmotility than are found by either procedure alone. Our findings indicate, however, that the determination of oesophageal transit time is at least as effective as manometry in the detection of oesophageal dysmotility. Being non-invasive and simple to perform, it is clearly a procedure of potential value in clinical practice.

\section{References}

1 Russell COH, Hill LD, Holmes ER, Hull DA, Gannon R, Pope CE. Radionuclide transit: a sensitive screening test for oesophageal dysfunction. Gastroenterology 1981; 80: 887-92.

2 Vantrappen G, Hellemans J. Diffuse muscle spasm of the oesophagus. Clin Gastroenterol 1976; 5 (1): 59-72.

3 Kazem I. A new scintigraphic technique for the study of the esophagus. AJR 1972; 115: 681-8.

4 Bosch A, Dietrich R, Lanaro A, Frias Z. Modified scintigraphic technique for the dynamic study of the oesophagus. Int J Nucl Med Biol 1977; 4: 195-9.

5 Mayron LW, Kaplan E. The use of ${ }^{81 \mathrm{~m}} \mathrm{Kr}$ in deglutinition kinetic studies Int J Nucl Med Biol 1975; 2 (1): 42-3.

6 Tolin RD, Malmud LS, Reilley J, Fisher RS. Esophageal scintigraphy to quantitate esophageal transit (quantitation of esophageal transit). Gastroenterology 1979; 76: 1402-8.

7 Fisher RS, Malmud LS, Roberts GS, Lobis IF. Gastroesophageal (GE) scintiscanning to detect and quantitate GE reflux. Gastroenterology 1976; 70: 301-8. 